Research Article

\title{
Dynamic Intelligent Feedback Scheduling in Networked Control Systems
}

\author{
Hui-ying Chen, Zu-xin Li, and Pei-liang Wang \\ School of Information \& Engineering, Huzhou Teachers College, Zhejiang 313000, China \\ Correspondence should be addressed to Hui-ying Chen; hychen@hutc.zj.cn
}

Received 11 September 2013; Accepted 14 October 2013

Academic Editor: Zhengguang Wu

Copyright (C) 2013 Hui-ying Chen et al. This is an open access article distributed under the Creative Commons Attribution License, which permits unrestricted use, distribution, and reproduction in any medium, provided the original work is properly cited.

\begin{abstract}
For the networked control system with limited bandwidth and flexible workload, a dynamic intelligent feedback scheduling strategy is proposed. Firstly, a monitor is used to acquire the current available network bandwidth. Then, the new available bandwidth in the next interval is predicted by using LS_SVM approach. At the same time, the dynamic performance indices of all control loops are obtained with a two-dimensional fuzzy logic modulator. Finally, the predicted network bandwidth is dynamically allocated by the bandwidth manager and the priority allocator in terms of the loops' dynamic performance indices. Simulation results show that the sampling periods and priorities of control loops are adjusted timely according to the network workload condition and the dynamic performance of control loops, which make the system running in the optimal state all the time.
\end{abstract}

\section{Introduction}

Although networked control systems (NCSs) have been widely used in numerous fields, the available network bandwidth resources of many control network applications are often limited due to economic or technical and other reasons [1]. For instance, CAN-bus can only provide at most $1 \mathrm{Mb} / \mathrm{s}$ transmission rate [2]; even if there exist network technologies with higher rate at present, their costs are relatively high. In addition, when several subsystems share the same communication network in the NCS, the number of network nodes is often variable along with the change of network user demands; at the same time, the disturbances of network environment or transmission of some aperiodic signals and other factors will lead to the variations of network workload. Therefore, for one or several specific control loops in the NCS, the available network resources of the system are not constant but are time-varying and nondeterministic. These above two factors will make the system incur some issues such as network-induced latency, data dropouts, and jitter, which will affect the system's performance and even lead to the instability of systems [3-8]. Thus, when the NCS with limited bandwidth is running in a workload flexible network environment, the overall control performance of the system not only depends on the design of control algorithm, but also depends on the scheduling and allocation of the available network bandwidth resources.

Until now, there are various scheduling methodologies for NCS with limited bandwidth resources [9-20]. The static scheduling strategies are traditionally employed as bandwidth scheduling techniques, as reported in [9-12] and the references therein. These bandwidth scheduling strategies allocated a fixed bandwidth to each control loop, allowing them to satisfy specified control performance requirements, and often work well in a given network environment. However, these static strategies may not be optimal allocation techniques for NCS with bandwidth constraints and cannot provide a desired control performance in an unpredictable open environment.

In recent years, researches on dynamic scheduling of NCS have received a great deal of attention and many results have been reported; see examples in [13-19]. In these dynamic scheduling strategies, the bandwidth resources are dynamically allocated by adjusting the sampling periods of control loops in runtime, so that the system can sufficiently utilize the sharing resources and effectively improve the overall control performance. In [13], Velasco adopted an augmenting statespace model and used an exponential function to dynamically allocate available bandwidth. In [14], considering single optimal objective of control performance subject to available 
bandwidth constraints, an optimal bandwidth allocation strategy based on linear programming technique was proposed. In [15], two intelligent and optimal scheduling strategies, based on fuzzy logic and neural network techniques, respectively, were employed for optimal bandwidth scheduling in multiloop NCS. Moreover, in [16], a network monitor was introduced to obtain the current network bandwidth and network-induced transfer error; subsequently, the sampling period of each control loop was dynamically regulated in terms of the estimated available network resources and network-induced error. However, these dynamic scheduling strategies work well only in a known workload.

However, real-world control applications sharing a communication network are usually suffering from the uncertainty of flexible network workload as mentioned above. To handle this issue, some feedback scheduling methodologies, such as neural network based feedback scheduling technique [8] and least squares support vector machines (LS_SVM) based feedback scheduling technique [21], have been presented to automate the management of flexible workload in NCS. In these methodologies, the network condition is predicted online by the feedback scheduler and then the control period is dynamically adjusted in response to estimated available network utilization. However, the above feedback scheduling strategies only discuss the dynamic bandwidth allocation of single control loop. To the best of our knowledge, few related results have been established for multiloop NCS with respect to bandwidth constraints and workload variations, which motivates the work of this paper.

In this paper, we present a novel dynamic feedback scheduling strategy for multiloop NCS with limited bandwidth and flexible workload existing in many control applications. In this work, the LS_SVM technique and fuzzy logic control technique are employed to construct a feedback scheduler that is able to handle the bandwidth constraints and workload variations intelligently.

This paper is organized as follows. The dynamic feedback scheduling architecture is given in Section 2. Section 3 states the involved algorithms in the feedback scheduler, including LS_SVM based available bandwidth prediction algorithm, fuzzy logic based system performance evaluation mechanism, bandwidth allocation algorithm, and priority assignment algorithm. Numerical simulations are given in Section 4 to evaluate the performance of the proposed method. Section 5 concludes this paper.

\section{Dynamic Feedback Scheduling Architecture}

2.1. System Architecture. The system considered in this work is shown in Figure 1. In this framework, there are $\mathrm{N}$ independent control loops that share a common communication network together with a dynamic feedback scheduler and other inference nodes. The workload within the network may vary in runtime, and hence the available network resources for this control application are time-varying and nondeterministic. In each control loop, the sensor node is time-driven and sends messages with a single packet according to the sampling period and the priority prespecified by the dynamic feedback scheduler, while the controller node and actuator node are event-driven. The dynamic feedback scheduler dynamically adjusts the sampling periods and priorities of control loops in accordance with the network condition and loops' dynamic control performance and works periodically in a time-triggered mode ( $T s$ is the incentive interval). The interference nodes in the system can also represent additional network workloads.

2.2. The Structure of Dynamic Feedback Scheduler. The structure of dynamic feedback scheduler is shown in Figure 2, which mainly consists of five components: a network monitor, an available bandwidth predictor, a fuzzy logic controller, a bandwidth manager, and a priority allocator. The main function of each component is as follows.

(1) The network monitor interfaces with the control network and measures the current quality of service (QoS) level of network at each incentive moment. The QoS level can be described by the available network utilization $d$, which directly reflects the uncertainty and flexibility of network bandwidth resources.

(2) The available bandwidth predictor based on LS_SVM technique is responsible for achieving the estimated value $\widehat{U}(j+1)$ of the available network utilization in the next incentive interval according to the current network utilization $d_{j}$ and some history data of network bandwidth. This enables the feedback scheduler to work in an intelligent way based on the history knowledge of the workload variations and to act in advance.

(3) The fuzzy logic controller, based on the error $e(j)$ and the difference of error $e c(j)$ of each control loop (as its two inputs), is used to produce an output value as the dynamic performance index to evaluate the control performance of each loop.

(4) The bandwidth manager dynamically allocates the available network bandwidth for each control loop based on the estimated value of available network utilization and the dynamic performance indices of control loops and determines the new sampling period of each control loop in the next incentive interval.

(5) Similarly, the priority allocator dynamically assigns the priority of each control loop in the next incentive interval in terms of the dynamic performance indices of control loops.

Based on this feedback scheduling mechanism, the system can achieve a desired control performance by adjusting the sampling period and priority of each control loop under the circumstances of network workload variations. In the following sections, we will present the involved algorithms of main components in the feedback scheduler.

\section{Algorithms Involved in the Feedback Scheduler}

3.1. Available Bandwidth Prediction Principle Based on LS_SVM. At present, SVM prediction algorithm has been 


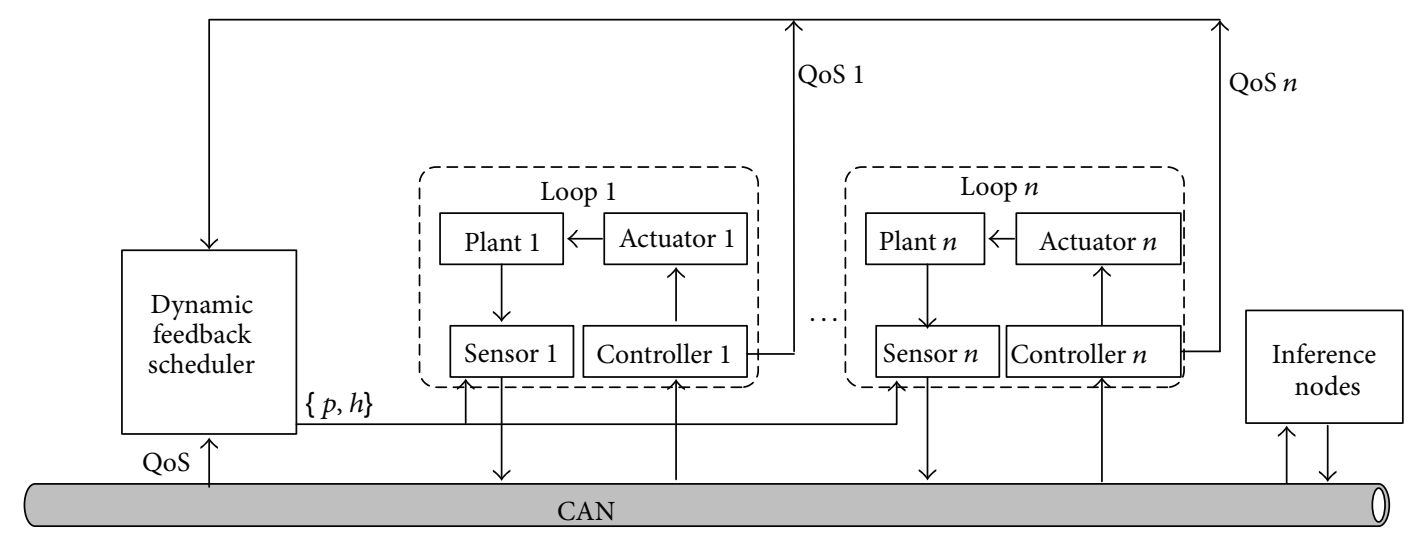

FIGURE 1: The networked control system structure with dynamic feedback scheduler.

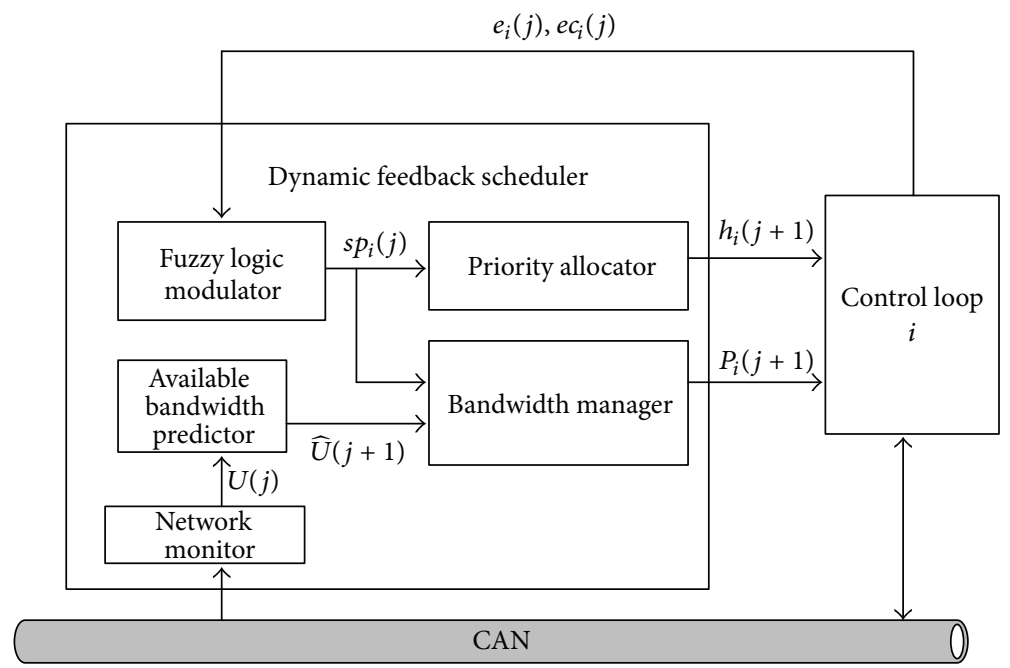

Figure 2: The structure of dynamic feedback scheduler.

widely used in pattern recognition, time series forecasting, and nonlinear modeling and identification [22]; moreover, it has been applied to time-delay prediction in NCS [23]. For the workload flexible network environment, the available network bandwidth can be seen as a time series with nonlinear characteristics, and we can use SVM technique for its online prediction.

3.1.1. SVM Prediction Model. In order to use SVM technique to conduct online prediction of the available bandwidth, first of all, the available bandwidth should be seen as a nonlinear time series and then should be reconstructed to establish a SVM-based prediction model.

Letting $d_{j}$ be the available network utilization monitored by the network monitor at the incentive moment $j$, we combined it with the available network utilization of the past incentive moments to constitute a time series of the available network utilization $D_{j}=\left\{d_{j-(N-1)}, \ldots, d_{j}\right\}$, and $L$ is the length of the time series. According to Takens' reconstruction theory of state space [24], at the incentive moment $j$, we can achieve the input and output training sample set of the SVM prediction model based on $D_{j}$ time series, respectively:

$$
\begin{aligned}
& {\left[\begin{array}{c}
x_{j-(M-1)} \\
x_{j-(M-2)} \\
\vdots \\
x_{j}
\end{array}\right]} \\
& =\left[\begin{array}{cccc}
d_{j-(L-1)} & d_{j-(L-1)+1} & \cdots & d_{j-(L-1)+m-2} \\
d_{j-(L-2)} & d_{j-(L-2)+1} & \cdots & d_{j-(L-2)+m-2} \\
\vdots & \vdots & \vdots & \vdots \\
d_{j-(L-M)} & d_{j-(L-M)+1} & \cdots & d_{j-1}
\end{array}\right] \text {, } \\
& {\left[\begin{array}{c}
y_{j-(M-1)} \\
y_{j-(M-2)} \\
\vdots \\
y_{j}
\end{array}\right]=\left[\begin{array}{c}
d_{j-(L-1)+m-1} \\
d_{j-(L-2)+m-1} \\
\vdots \\
d_{j}
\end{array}\right]}
\end{aligned}
$$


where $M$ is the number of observational sample, $m$ is an embedding dimension, $M=L-(m-1), x_{i} \in R^{m-1}, y_{i} \in R$, and $i=j-(M-1), \ldots, j$.

Within the incentive interval $j$, the prediction model based on SVM can be expressed as

$$
y_{i}=F\left(x_{i}\right) \quad i=j-(M-1), \ldots, j,
$$

where $F(g)$ is a required reconstructive nonlinear function, which needs to regress based on history data before the incentive moment $j$. According to the statistics theory, the fitting function of the available network utilization time series based on SVM can be defined as

$$
y=\sum_{i=j-(M-1)}^{j} \alpha_{i} K\left(x_{i}, x\right)+b
$$

where $\alpha_{i}(i=j-(M-1), \ldots, j)$ is the weighting coefficient of supporting vector, $b$ is the bias, and $K\left(x_{i}, x_{l}\right)$ is the kernel function, and there are several available kernel functions. Here, we employ the commonly used radial basis function $(\mathrm{RBF})$ as the kernel function:

$$
K\left(x_{i}, x_{l}\right)=\exp \left(-\frac{\left\|x_{i}-x_{l}\right\|^{2}}{2 \sigma^{2}}\right),
$$

where $\sigma$ is a hyperparameter of the kernel function and is a prespecified constant.

Thus, the available bandwidth prediction model based on SVM has been established. Next, we will use SVM to train the above constructed input and output training sample set and obtain the parameters $\alpha_{i}$ and $b$ in the prediction model. Then, we can predict the available network utilization of the next incentive interval according to (3).

3.1.2. Online LS_SVM Prediction. Since the normal SVM requires solving the convex quadratic programming problem, it is not conducive to the online prediction due to large amounts of calculation. While the least square support vector machine (LS_SVM) converts the inequality constraints of the normal SVM into equality constraints and the empirical risk from the first power of the deviation to the second power, thus, the rate of convergence is greatly improved by solving linear functions instead of quadratic programming in SVM.

At the incentive moment $j$, for a given training set $\left(x_{i}, y_{i}\right)$, $x_{i} \in R^{m-1}, y_{i} \in R$, and $i=j-(M-1), \ldots, j$, and according to structural risk minimization principle, the sample's least squares support vector machine regression problem can be defined as an optimization problem:

$$
\begin{aligned}
& \min _{\omega, b, e} J=\frac{1}{2} \omega^{T} \omega+C \sum_{i=j-(M-1)}^{j} e_{i}^{2}, \\
& \text { s.t. } \quad y_{i}=\omega^{T} \phi\left(x_{i}\right)+b+e_{i},
\end{aligned}
$$

where $\omega$ is a weighting vector, $C$ is a regularization parameter, and $\phi(\bullet)$ is a nonlinear function which maps the input space to a higher dimensional feature space. In order to solve the optimization problem, define the Lagrange function as

$$
\begin{aligned}
L= & \frac{1}{2} \omega^{T} \omega+C \sum_{i=j-(M-1)}^{j} e_{i}^{2} \\
& -\sum_{i=j-(M-1)}^{j} \alpha_{i}\left[\omega^{T} \phi\left(x_{i}\right)+b+e_{i}-y_{i}\right],
\end{aligned}
$$

where $\alpha_{i}$ is the multiplier of Lagrange. According to the Karush-Kuhn-Tucker (KKT) optimization condition, the following linear equation holds:

$$
\begin{aligned}
& {\left[\begin{array}{cccc}
0 & 1 & \cdots & 1 \\
1 & K\left(x_{j-(M-1)}, x_{j-(M-1)}\right)+\frac{1}{C} & \cdots & d_{j-(L-2)+m-2} \\
\vdots & \vdots & \vdots & \vdots \\
1 & d_{j-(L-M)+1} & \cdots & K\left(x_{j}, x_{j}\right)+\frac{1}{C}
\end{array}\right]} \\
& \times\left[\begin{array}{c}
b \\
\alpha_{j-(M-1)} \\
\vdots \\
\alpha_{j}
\end{array}\right]=\left[\begin{array}{c}
0 \\
y_{j-(M-1)} \\
\vdots \\
y_{j}
\end{array}\right] \text {, }
\end{aligned}
$$

where $K\left(x_{i}, x_{l}\right)=\left\langle\phi\left(x_{i}\right), \phi\left(x_{l}\right)\right\rangle$ is a kernel function, which satisfies Mercer condition, and the radial basis function (RBF) shown in (4) is employed.

By solving the above linear equation, we can obtain the regression coefficients $\alpha_{i}(i=j-(M-1), \ldots, j)$ and bias $b$ at the incentive moment $j$. At the same time, letting $x_{j+1}=$ $\left[d_{j-(L-M)+1}, \ldots, d_{j-1}, d_{j}\right]$, the prediction value of the available bandwidth in the incentive interval $j+1$ can be obtained:

$$
\widehat{U}(j+1)=F\left(x_{j+1}\right)=\sum_{i=j-(M-1)}^{j} \alpha_{i} K\left(x_{i}, x_{j+1}\right)+b .
$$

3.2. System Performance Evaluation Based on Fuzzy Logic. Among the dynamic scheduling research results in NCS, the network bandwidth or the priority of the system is often dynamically allocated and regulated according to the control performance of control loops, and the poorer the loop's performance is, the more bandwidth or the higher priority is allocated. To evaluate Quality of control (QoC) level of a control loop, we usually choose a performance index to measure, such as the instantaneous error and the integral absolute error (IAE) of control loop [18, 20].

Considering the case that the difference of error is big when the instantaneous error is through zero, for this case, if we directly select the instantaneous error as the control loop's performance index, it cannot reflect the real dynamic performance of system. Therefore, in order to better reflect the control loop's dynamic performance, we design a novel performance evaluation mechanism based on the fuzzy logic control technique. The main role of the fuzzy logic modulator is to produce an output value for each control loop as the 
dynamic performance index to characterize its QoC level. In this paper, a two-dimensional fuzzy logic modulator is designed, which has two input variables, the error $e_{i}(j)=$ $e_{i}(k)$ and the difference of error $e c_{i}(j)=e_{i}(k)-e_{i}(k-1)$. These two inputs can reflect the degree of deviation from system's equilibrium and the trend of system's trajectory, respectively. The modulator is composed of four main components: a fuzzifier, a rule base, an inference mechanism, and a defuzzifier. Once activated at the incentive moment $j$, for the control loop $i$, the fuzzifier translates numeric input variables $e_{i}(j)$ and $e c_{i}(j)$ into fuzzy sets characterizing linguistic variables $E$ and $E C$. The inference mechanism then applies a predetermined set of linguistic rules in the rule base with respect to these linguistic variables and produces the fuzzy sets of the output linguistic variable $S P$. In this work, the linguistic values for input variables of $E$ and $E C$ are NB (negative big), NS (negative small), ZE (zero), PS (positive small), and PB (positive big). The linguistic values of output variable SP are VS (very small), S (small), SM (small medium), BM (big medium), B (big), and VB (very big). Figure 3 depicts the membership functions of input variables $E, E C$ and output variable $S P$. Finally, the defuzzifier converts the fuzzy conclusions reached by the inference mechanism to a numeric value $s p_{i}(j)$.

Through analysis of the running conditions of system under different states and combining with the knowledge of relevant control theory, 25 fuzzy rules are built as shown in Table 1. The main idea of establishing the fuzzy logic rule is; when the error and the difference of error in the control loop are paralleling (both positive or both negative) and their absolute values are big, it means that the system's trajectory will keep away from equilibrium and also means that the loop's performance is poor, so the dynamic performance index should be made big. When the error and the difference of error in the control loop are small, it means that the system's trajectory is close to equilibrium and also means that the loop's performance is good, so the dynamic performance index should be made small. When the error and the difference of error in the control loop are reverse, it means the system's trajectory is nearing to equilibrium and also means that the loop's performance develops in the right direction, so the loop's performance index can be made relatively small.

In the fuzzy inference mechanism, Max-Min (maximumminimum) reasoning method is employed. The weighting average method is used in the defuzzification to calculate the numeric value $s p_{i}$, and $s p_{i}$ is chosen to be $[0.011]$. The closer its value is to 0.01 , the better the loop's performance is. Conversely, the closer its value is to 1 , the worse the loop's performance is. In addition, in this paper, the lower limit set to 0.01 , but not zero, is mainly to avoid the denominator being zero in the following bandwidth allocation algorithm.

The loop's dynamic performance index obtained by the above algorithm, fully considering the error and the difference of error in the control loop, can better reflect the dynamic performance of control loop. In addition, in the design of fuzzy logic modulator, we can make a query table beforehand according to the above algorithm and adopt the lookup table method to reduce computational complexity in the fuzzy reasoning.
TABLE 1: Fuzzy rule base.

\begin{tabular}{lccccc}
\hline SP & & & $E C$ & & \\
& NB & NS & ZE & PS & PB \\
\hline$E$ & & & & & \\
NB & VB & VB & VB & VB & B \\
NS & B & BM & SM & SM & SM \\
ZE & SM & S & VS & S & SM \\
PS & SM & SM & SM & BM & B \\
PB & B & VB & VB & VB & VB \\
\hline
\end{tabular}
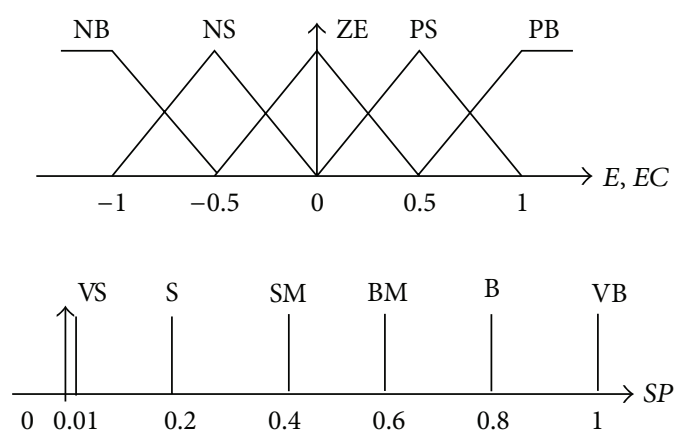

FIGURE 3: Membership functions of input variables $E, E C$, and output variable $S P$.

3.3. Bandwidth Allocation Algorithm. The bandwidth manager dynamically allocates the available bandwidth of each control loop based on the estimated value of the available network utilization and the dynamic performance indices of control loops to optimize the overall control performance of the system. The basic principle of allocation is, the poorer the dynamic performance of control loop is, the more available bandwidth is assigned. In other words, the better the dynamic performance of control loop is, the less available bandwidth is assigned.

Take the output value of the fuzzy logic modulator as an index to characterize the QoC level of control loop; that is,

$$
J_{i}(j)=s p_{i}(j)
$$

where $s p_{i}$ is the fuzzy logic output value of control loop $i$.

According to (9) and considering the different conditions of the evaluated value of available network utilization, the bandwidth allocation algorithm is designed as follows.

(1) When $\widehat{U}(j)>\sum\left(c_{i} / h_{i, \max }\right)$,

$$
\begin{aligned}
\widehat{U}_{i}(j) & =U_{i, \min }+\left(\widehat{U}(j)-\sum U_{i, \min }\right) \frac{w_{i} J_{i}(j)}{\sum w_{i} J_{i}(j)} \\
& =\frac{c_{i}}{h_{i, \max }}+\left(\widehat{U}(j)-\sum \frac{c_{i}}{h_{i, \max }}\right) \frac{w_{i} J_{i}(j)}{\sum w_{i} J_{i}(j)},
\end{aligned}
$$

where $U_{i, \min }=c_{i} / h_{i, \max }$ is the minimum available utilization of control loop $i$ and $w_{i}$ is the weighting coefficient.

(2) When $\widehat{U}(j) \leq \sum\left(c_{i} / h_{i, \max }\right)$,

$$
\widehat{U}_{i}(j)=U_{i, \min } \cdot
$$


From (10), it can be seen that the allocation of bandwidth is on basis of guaranteeing the minimum available bandwidth for each control loop firstly and then distributes the rest of the bandwidth resources in proportion according to the value of $w_{i} J_{i}$. In this way, the control loop with poorer performance will get more available network bandwidth, while the control loop with better performance will be allocated less bandwidth resources. Such bandwidth allocation strategy can optimize the utilization of network resources and improve the overall control performance of the system.

From the definition of the network bandwidth, we can obtain the sampling period of each control loop within the incentive interval $j[20]$ :

$$
h_{i}(j)=\frac{c_{i}}{\widehat{U}_{i}(j)}, \quad i=1, \ldots N .
$$

Furthermore, in order to reduce the sampling period jitter of each control loop, we can set a sampling period threshold $\Delta h$, and the control period will be refreshed only when the sampling period meets $\left|h_{i}(j+1)-h_{i}(j)\right|>\Delta h$.

3.4. Priority Assignment Algorithm. When the network resources are limited, especially when the network workload is overloaded, the data of control loop with lower priority cannot be updated for a long time, so that the control loop will be seriously affected by packet loss and long latency, which result in the control performance deterioration and even instability. Therefore, in order to optimize the overall control performance of the system, it is crucial to dynamically assign the priority of control loop. Reference $[25,26]$ employed the MEF method with the main idea of dynamically distributing the priority of control loop based on its error value; and that the larger the error is, the higher the loop's priority is. Similar to the bandwidth allocation algorithm, in this paper, the control loop performance index $J_{i}^{\prime}(j)=w_{i} J_{i}(j)$ is selected for priority assignment, and the definition of $w_{i}$ and $J_{i}(j)$ is the same as the above. Based on the dynamic performance index, the assignment principle of loop's priority is, within each incentive interval, the priority $P_{i}(j)$ of control loop $i$ is set according to the value of $J_{i}^{\prime}(j)$, and the larger the $J_{i}^{\prime}(j)$ is, the higher the loop's priority is. For the control loops with the same $J_{i}^{\prime}(j)$, they will maintain the priority level of the previous incentive interval.

\section{Performance Evaluation}

4.1. Simulation Setup. In this section, considering two servo control systems through a CAN-bus, the plant model is given as follows:

$$
\begin{gathered}
\dot{x}=\left[\begin{array}{cc}
-20 & 0 \\
1 & 0
\end{array}\right] x+\left[\begin{array}{c}
540 \\
0
\end{array}\right] u, \\
y=\left[\begin{array}{ll}
0 & 1
\end{array}\right] x .
\end{gathered}
$$

The feedback controller $u=-K x$ is designed by pole placement approach without network, and we obtain $K=$ [0.0185 0.8333]. Assume that the initial sampling periods of the two control loops are $h_{1}=10 \mathrm{~ms}$ and $h_{2}=12 \mathrm{~ms}$,

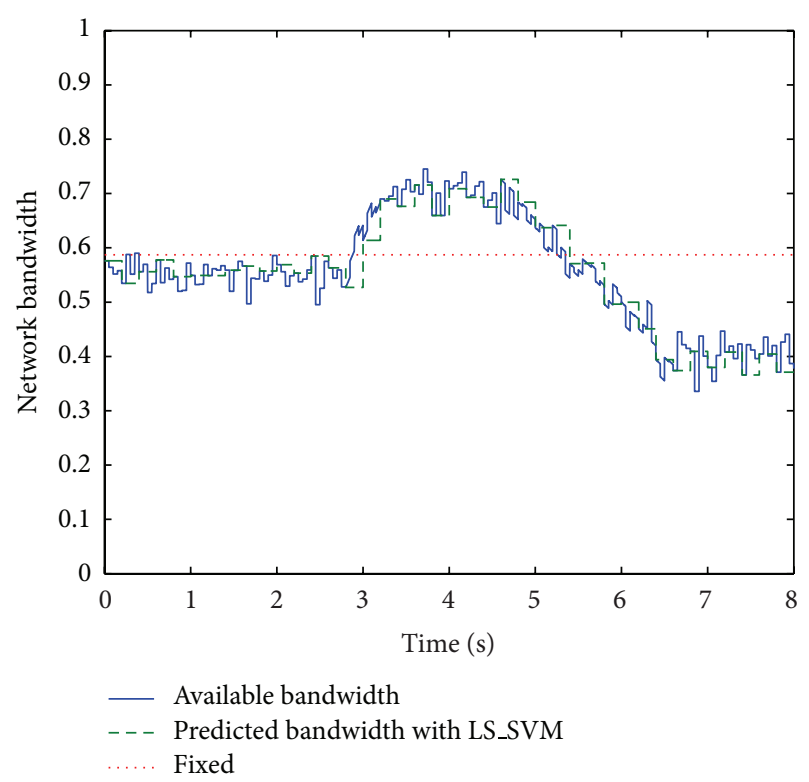

(a) The measure and prediction of network utilization

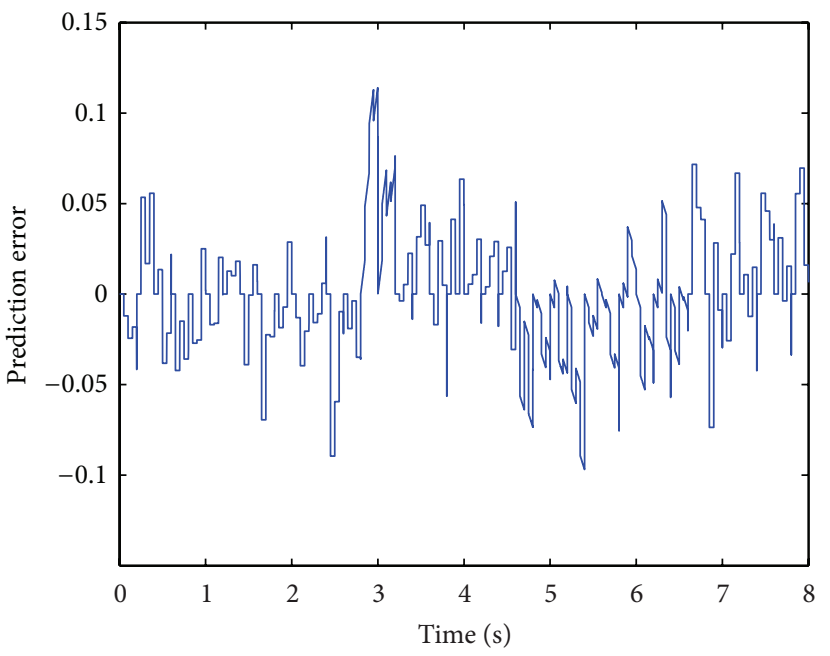

(b) The prediction error

FIGURE 4: The network utilization prediction based on LS_SVM.

respectively; the first control loop has a higher priority, and all the weighting coefficients of control loops are $\omega_{i}=1$. The data rate of CAN-bus is $25 \mathrm{kbps}$, and the size of the sampled data is 10 bytes. In addition, the parameters of dynamic feedback scheduler are shown in Table 2.

In order to illustrate the superiority of the proposed method (dynamic feedback scheduling allocation, DFSA) under the network environment with limited bandwidth and flexible workload, we compare it with another scheduling strategy (error-based dynamic feedback allocation [20], EDFA, in which the network bandwidth and priority are dynamically allocated according to the errors of control loops), and the simulation is implemented based on Matlab/TrueTime. 


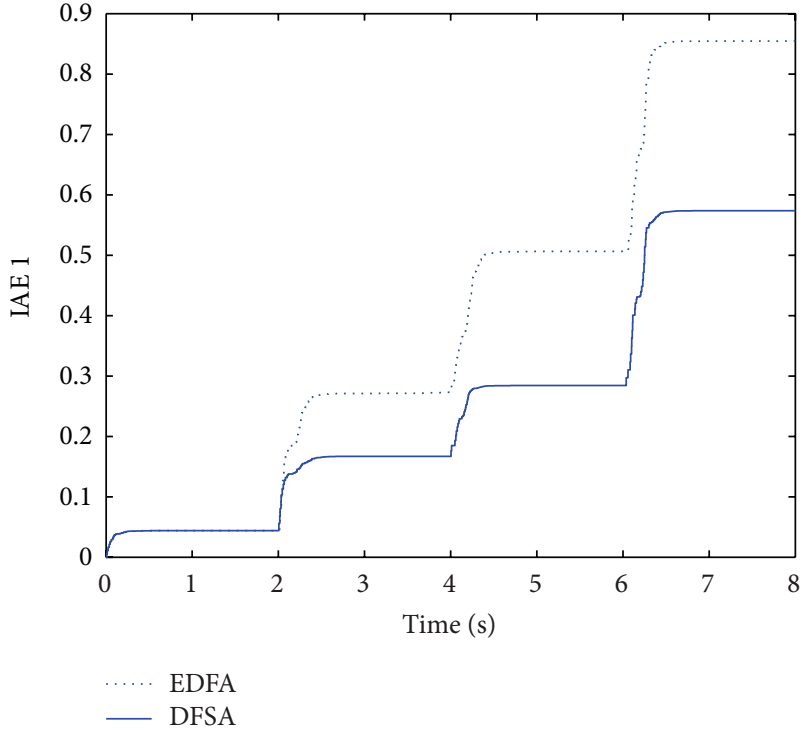

(a) IAE performance of control loop 1

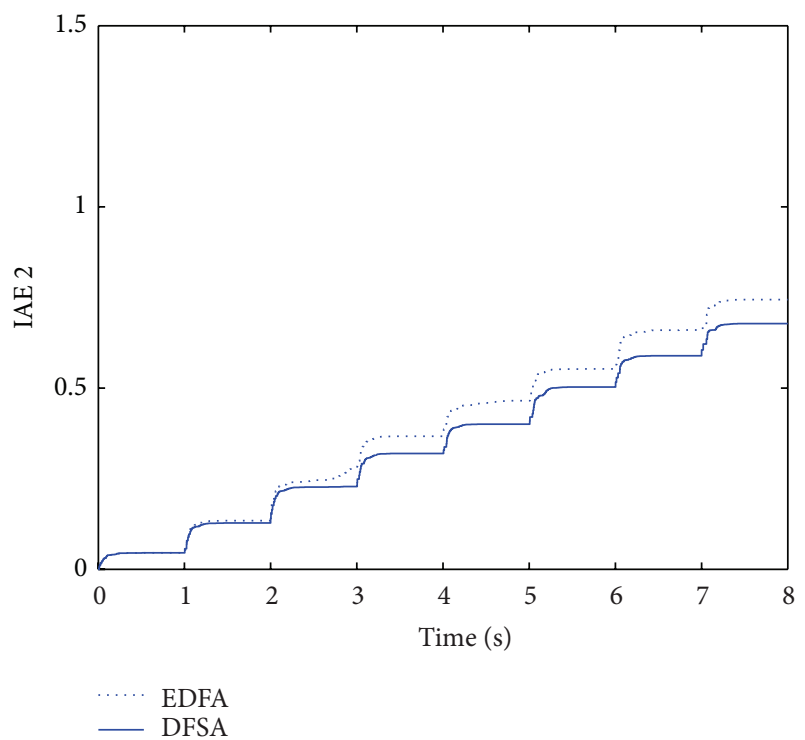

(b) IAE performance of control loop 2

FIGURE 5: IAE performance of the system.

4.2. Results. When the feedback scheduler is activated periodically, the available network utilization can be measured by the network monitor. The measured value and its prediction value are shown in Figure 4(a), and the prediction error is shown in Figure 4(b). Due to workload variations, in the three intervals, the network bandwidth fluctuates around $0.57,0.7$, and 0.4 , respectively. It can be easily seen from Figure 4(a) that the prediction algorithm based on LS_SVM always accurately forecasts the new network utilization of the next incentive interval. From Figure 4(b), we know that the prediction error is roughly within the range of \pm 0.05 , but it increases at the volatile moment of network utilization.

In this paper, IAE (integral absolute error) is chosen as the overall control performance index of control loop; the IAE

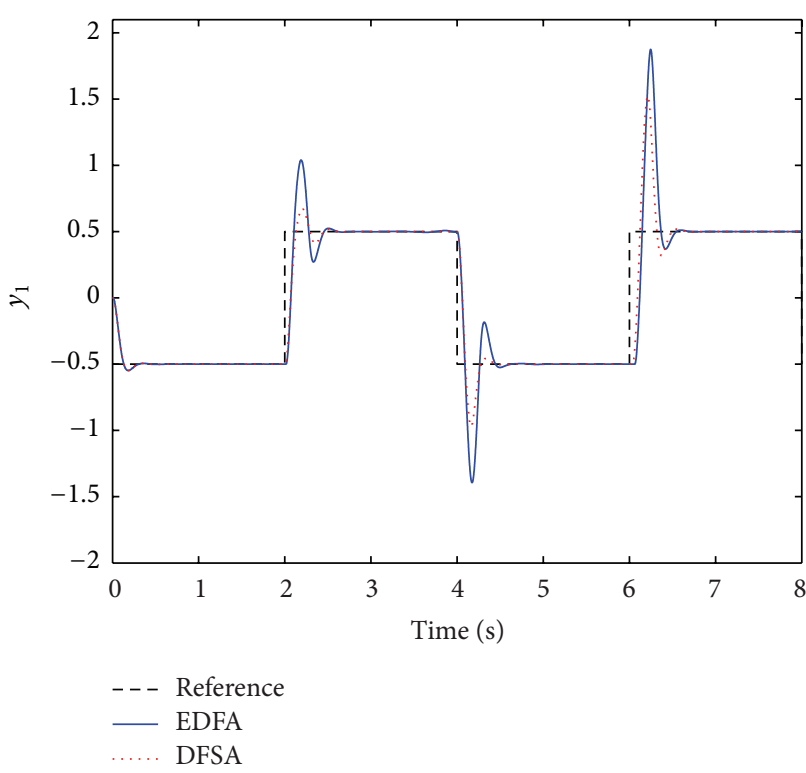

(a) The response curve of control loop 1

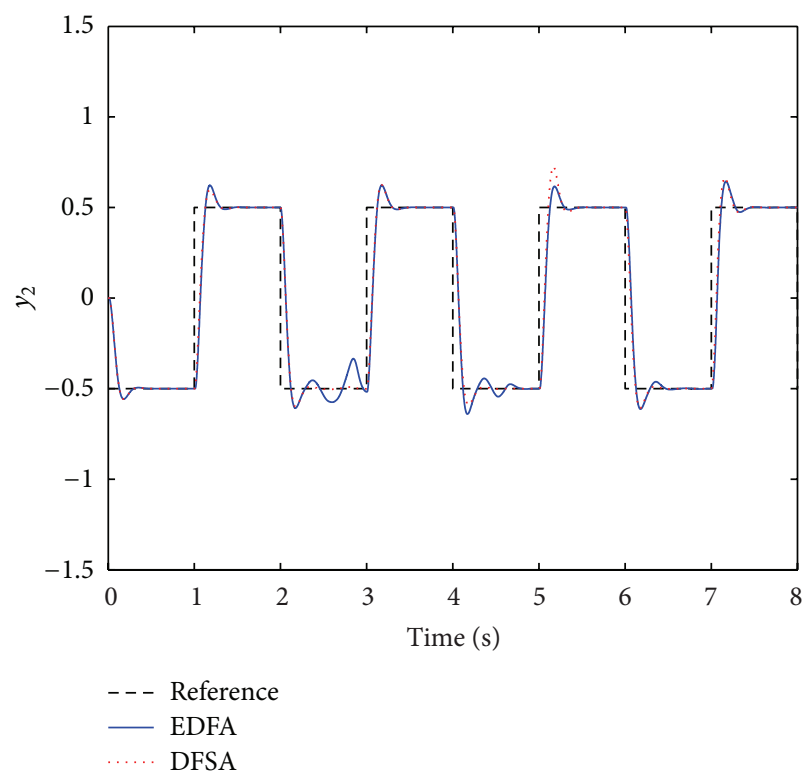

(b) The response curve of control loop 2

Figure 6: The response curves of the system.

TABLE 2: Simulation parameters of dynamic feedback scheduler.

\begin{tabular}{lccccccc}
\hline Parameter & $T s$ & $h_{i, \max }$ & $L$ & $m$ & $\sigma$ & $C$ & $\Delta h$ \\
\hline Value & $200 \mathrm{~ms}$ & $20 \mathrm{~ms}$ & 5 & 4 & 0.96 & 3.3 & 0.001 \\
\hline
\end{tabular}

curves and the response curves of the system are shown in Figures 5 and 6 , respectively. It can be easily seen that under the network environment with limited bandwidth and flexible workload, the system has always remained stable through dynamically adjusting the sampling period and priority of each control loop, and the overall control performance of the system obtained by the proposed scheduling algorithm in this work is superior to EDFA. 


\section{Conclusions}

Control applications are usually running in a communication network with bandwidth constraints and unpredictable open environment. In this paper, an intelligent dynamic feedback scheduling strategy is proposed for multiloop NCS with limited bandwidth and flexible workload. In order to handle uncertain and flexible workload in the control networks, we introduce an online LS_SVM approach to predict the available network bandwidth, which is then dynamically allocated by the bandwidth manager and the priority allocator in terms of the dynamic performance indices of control loops. Different from the existing bandwidth allocation and priority assignment strategies, we introduce a novel control performance evaluation mechanism based on fuzzy logic control technique. The simulation results demonstrate that the proposed strategy can improve the overall control performance to the maximum and allow control applications to have more flexibility in the uncertain running conditions.

\section{Acknowledgments}

The authors thank the reviewers for their very helpful comments and suggestions, which have improved the presentation of this paper. This study is supported by the National Natural Science Foundation of China under Grant no. 61174029, the Key Project of Chinese Ministry of Education under Grant no. 211067, and the Zhejiang Provincial Natural Science Foundation of China under Grant no. LY12F03008.

\section{References}

[1] P. Antsaklis and J. Baillieul, "Special issue on networked control systems," IEEE Transactions on Automatic Control, vol. 49, no. 9, pp. 1421-1423, 2004.

[2] Bosch, "CAN specification (version 2.0)," 1991.

[3] R. A. Gupta and M.-Y. Chow, "Networked control system: overview and research trends," IEEE Transactions on Industrial Electronics, vol. 57, no. 7, pp. 2527-2535, 2010.

[4] Z. W. Wang and H. T. Sun, "Control and scheduling co-design of networked control system: overview and directions," in Proceedings of the International Conference on Machine Learning and Cybernetics, pp. 816-824, Xian, China, 2012.

[5] L. X. Zhang, H. J. Gao, and O. Kaynak, "Network-induced constraints in networked control systems-a survey," IEEE Transactions on Industrial Informatics, vol. 9, no. 1, pp. 403-416, 2013.

[6] Z. G. Wu, P. Shi, H. Su, and J. Chu, "Exponential synchronization of neural networks with discrete and distributed delays under time-varying sampling," IEEE Transactions on Neural Networks and Learning Systems, vol. 23, no. 9, pp. 1368-1376, 2012.

[7] Z. G. Wu, P. Shi, H. Su, and J. Chu, "Stochastic synchronization of Markovian jump neural networks with time-varying delay using sampled-data," IEEE Transactions on Cybernetics, pp. 111, 2013.

[8] F. Xia, S. B. Li, and Y. X. Sun, "A Neural network based feedback scheduler for networked control system with flexible workload," Lecture Notes in Computer Science, vol. 3611, pp. 242-251, 2005.
[9] S. H. Hong, "Bandwidth allocation scheme for cyclic-service fieldbus networks," IEEE/ASME Transactions on Mechatronics, vol. 6, no. 2, pp. 197-204, 2001.

[10] H. S. Park, Y. H. Kim, D.-S. Kim, and W. H. Kwon, "A scheduling method for network-based control systems," IEEE Transactions on Control Systems Technology, vol. 10, no. 3, pp. 318-330, 2002.

[11] M. S. Branicky, S. M. Phillips, and W. Zhang, "Scheduling and feedback co-design for networked control systems," in Proceedings of the 41st IEEE Conference on Decision and Control, pp. 1211-1217, Las Vegas, Nev, USA, December 2002.

[12] J. Q. He and H. C. Zhang, "The study on scheduling optimization in a network control system based on genetic algorithms," Industrial Instrumentation \& Automation, vol. 36, no. 4, pp. 3739, 2004.

[13] M. Velasco, J. M. Fuertes, C. Lin, P. Martí, and S. Brandt, "A control approach to bandwidth management in networked control systems," in Proceedings of the 30th Annual Conference of the IEEE Industrial Electronics Society, pp. 2343-2348, Busan, Korea, November 2004.

[14] M. Velasco, P. Martí, and M. Frigola, "Bandwidth management for distributed control of highly articulated robots," in Proceedings of the IEEE International Conference on Robotics and Automation, pp. 265-270, Barcelona, Spain, April 2005.

[15] Z. Li, W. Wang, and Y. Jiang, "Intelligent scheduling and optimisation for resource-constrained networks," IET Control Theory and Applications, vol. 5, no. 12, pp. 2982-2992, 2010.

[16] Y. Wang, H. Cai, Q.-W. Chen, and W.-L. Hu, "Feedback scheduler design of networked control systems," Acta Electronica Sinica, vol. 35, no. 2, pp. 379-384, 2007.

[17] F. Xia, Y. X. Sun, and Y. C. Tian, "Fuzzy feedback scheduling of resource-constrained embedded control systems," International Journal of Innovative Computing, Information and Control, vol. 5, no. 2, pp. 311-321, 2009.

[18] C. Peng, D. Yue, Z. Gu, and F. Xia, "Sampling period scheduling of networked control systems with multiple-control loops," Mathematics and Computers in Simulation, vol. 79, no. 5, pp. 1502-1511, 2009.

[19] Z. D. Tian, X. W. Gao, M. H. Shi, and K. Li, "Fuzzy feedback scheduler of resource constrained networked control system," Electronic Machine and Control, vol. 17, no. 1, pp. 94-101, 2013.

[20] F. Xia, Feedback Scheduling of Real-Time Control Systems with Resource Constraints, Zhejiang University, Hangzhou, China, 2006.

[21] Z.-X. Li, W.-L. Wang, and W.-J. Hu, "Least squares support vector machines based predictive feedback scheduling for resource-constrained networks," Control and Decision, vol. 25, no. 3, pp. 361-366, 2010.

[22] Z.-J. Lu, J.-G. Yang, Q. Xiang, and X.-L. Wang, "Research on support vector machines based predictive model for yarn quality," Control and Decision, vol. 22, no. 6, pp. 693-696, 2007.

[23] C.-M. Li, J. Xiao, and Y. Zhang, "Approach of adaptive prediction control on networked control systems based on leastsquares support vector machines," Journal of System Simulation, vol. 19, no. 15, pp. 3494-3502, 2007.

[24] F. Takens, "Detecting strange attractors in fluid turbulence," in Dynamical Systems and Turbulence, pp. 366-381, Springer, Belin, Germany, 1981.

[25] J. Yépez, P. Martí, and J. M. Fuertes, "Control loop scheduling paradigm in distributed control systems," in Proceedings of the 29th Annual Conference of the IEEE Industrial Electronics Society, pp. 1441-1446, Roanoke, Va, USA, November 2003. 
[26] F. Xia, Z. Wang, and Y. Sun, "Simulation based performance analysis of networked control systems with resource constraints," in Proceedings of the 30th Annual Conference of the IEEE Industry Electronics Society, pp. 2946-2951, Busan, Korea, November 2004. 


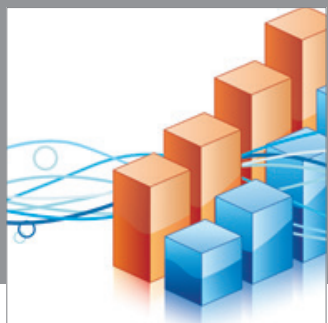

Advances in

Operations Research

mansans

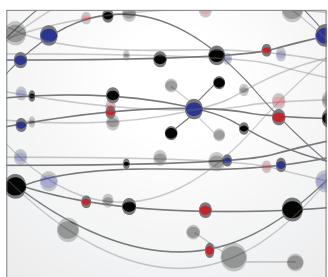

The Scientific World Journal
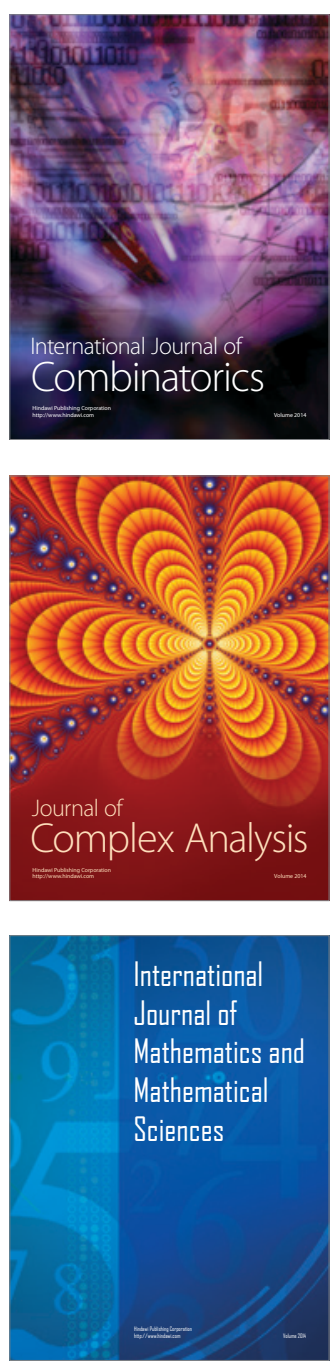
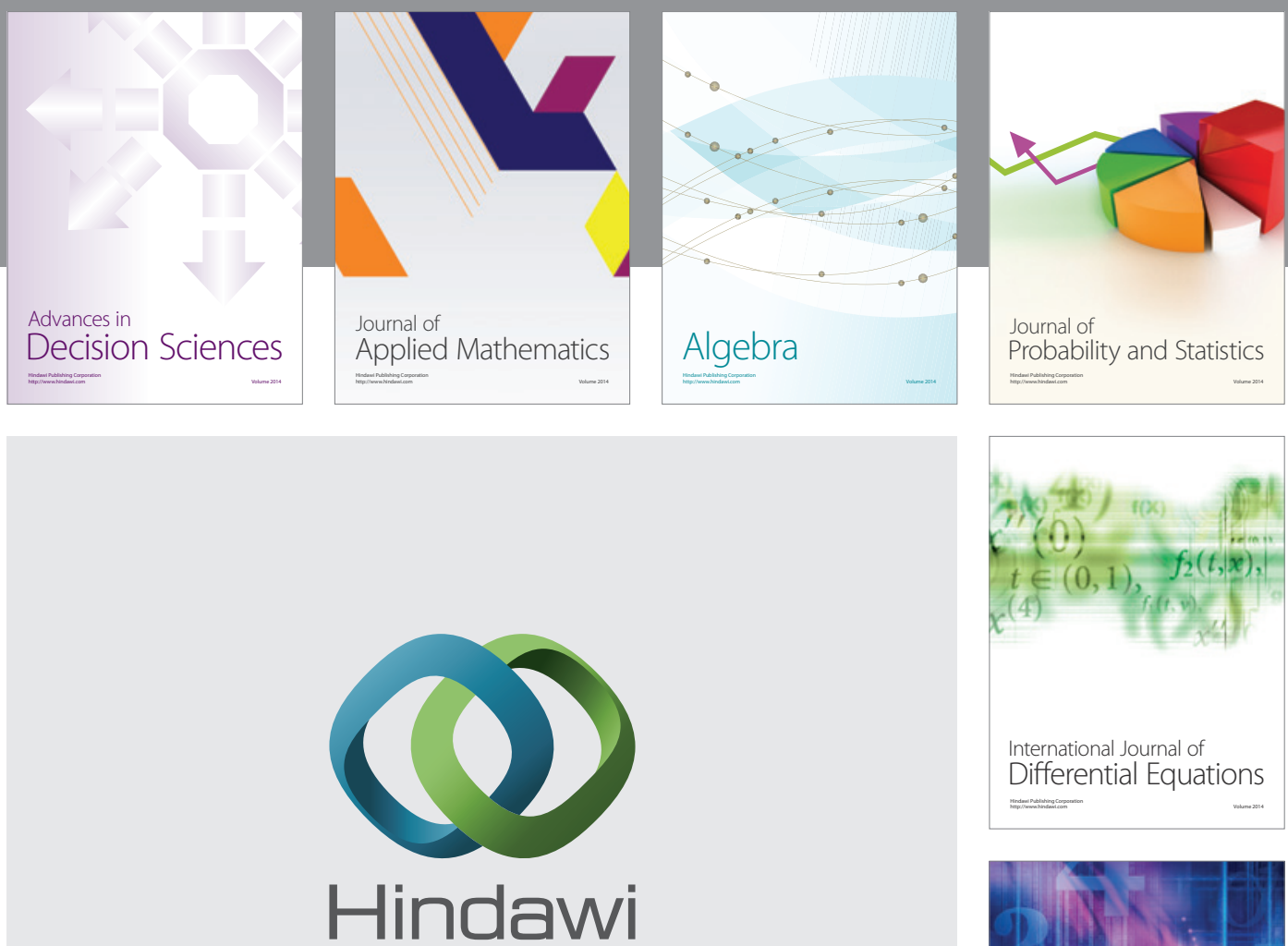

Submit your manuscripts at http://www.hindawi.com
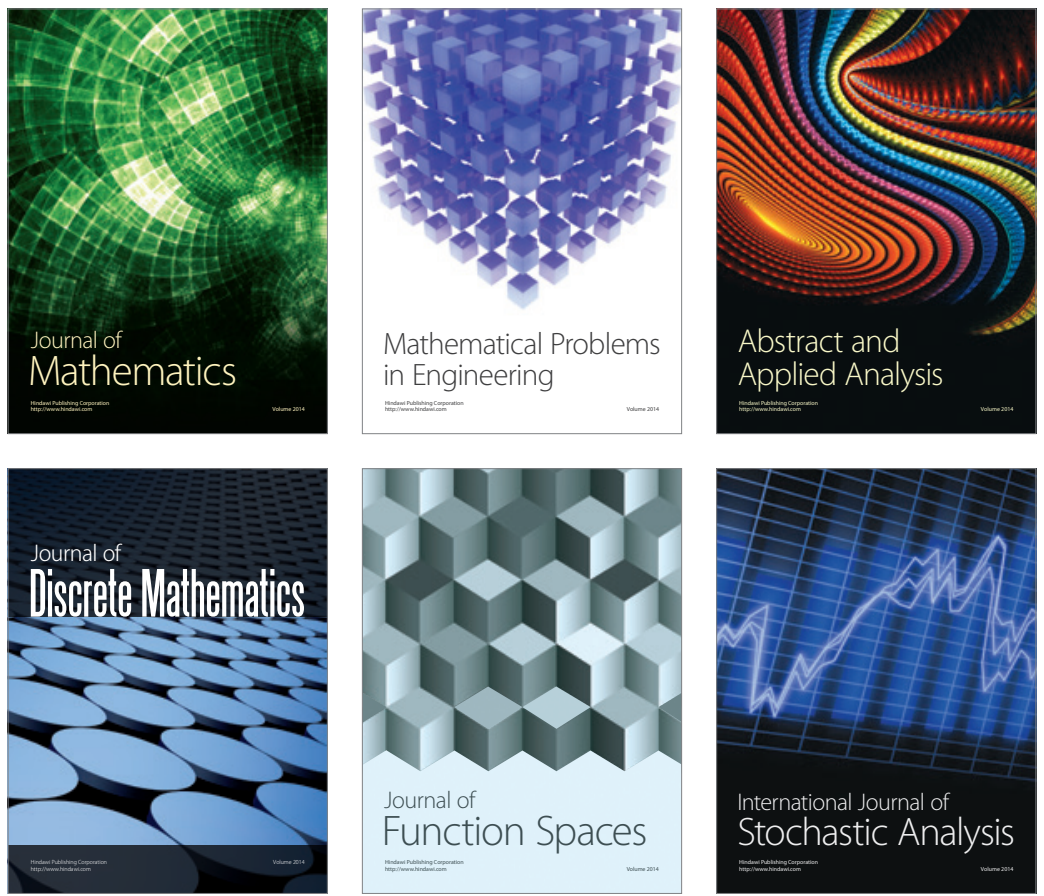

Journal of

Function Spaces

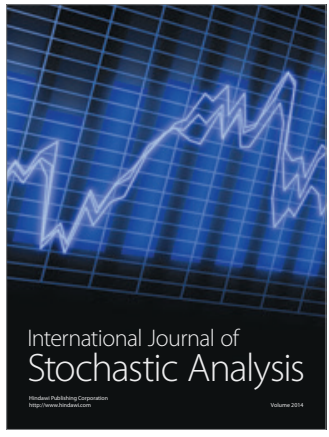

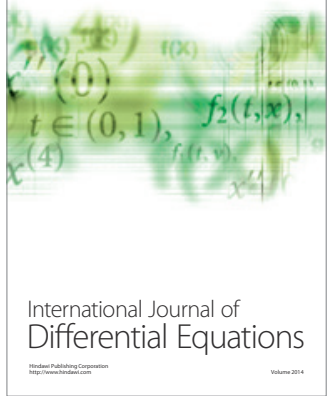
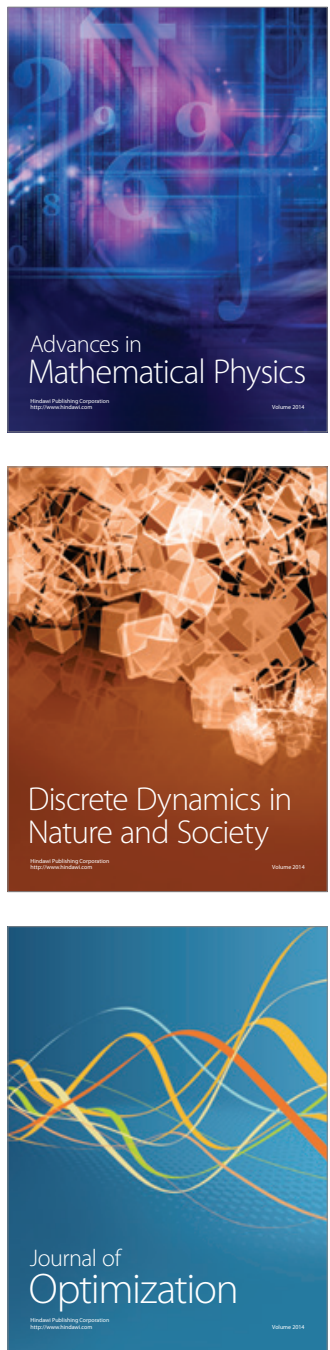